\title{
Characterisation of microbial communities within aggressive prostate cancer tissues
}

\author{
Melissa A. Yow ${ }^{1}$, Sepehr N. Tabrizi ${ }^{2,3,4}$, Gianluca Severi ${ }^{9}$, Damien M. Bolton ${ }^{7}$, John Pedersen ${ }^{8}$, Australian Prostate \\ Cancer BioResource $^{10}$, Graham G. Giles ${ }^{5,6}$ and Melissa C. Southey ${ }^{1 *}$
}

\begin{abstract}
Background: An infectious aetiology for prostate cancer has been conjectured for decades but the evidence gained from questionnaire-based and sero-epidemiological studies is weak and inconsistent, and a causal association with any infectious agent is not established. We describe and evaluate the application of new technology to detect bacterial and viral agents in high-grade prostate cancer tissues. The potential of targeted 16S rRNA gene sequencing and total RNA sequencing was evaluated in terms of its utility to characterise microbial communities within high-grade prostate tumours.

Methods: Two different Massively Parallel Sequencing (MPS) approaches were applied. First, to capture and enrich for possible bacterial species, targeted-MPS of the V2-V3 hypervariable regions of the 16S rRNA gene was performed on DNA extracted from 20 snap-frozen prostate tissue cores from ten "aggressive" prostate cancer cases. Second, total RNA extracted from the same prostate tissue samples was also sequenced to capture the sequence profile of both bacterial and viral transcripts present.

Results: Overall, $16 \mathrm{~S}$ rRNA sequencing identified Enterobacteriaceae species common to all samples and P. acnes in 95\% of analyzed samples. Total RNA sequencing detected endogenous retroviruses providing proof of concept but there was no evidence of bacterial or viral transcripts suggesting active infection, although it does not rule out a previous 'hit and run' scenario.

Conclusions: As these new investigative methods and protocols become more refined, MPS approaches may be found to have significant utility in identifying potential pathogens involved in disease aetiology. Further studies, specifically designed to detect associations between the disease phenotype and aetiological agents, are required.
\end{abstract}

Keywords: Prostate cancer, Sexually transmitted infection, Infection, $16 \mathrm{~S}$ rRNA, RNA, cDNA, Propionibacterium acnes

\section{Background}

First proposed in the early 1950s, an infectious aetiology for prostate cancer has since been widely investigated using conventional and serology-based case-control designs and some cohort studies but the evidence from these has been generally weak and inconsistent. A causal association is yet to be established.

Recent support for a role of infection in prostate cancer risk came from the detection of a novel candidate, Propionibacterium acnes, within prostate cancer tissues $[1,2]$. There is also evidence of association between prostate cancer risk and gene variants of COX-2 [3], RNASEL [4] and TLR4 [5], identified in cases of hereditary

\footnotetext{
* Correspondence: msouthey@unimelb.edu.au

${ }^{1}$ Genetic Epidemiology Laboratory, Department of Pathology, Faculty of Medicine, Dentistry and Health Sciences, University of Melbourne, VIC, Australia 3010

Full list of author information is available at the end of the article
}

prostate cancer, indicating that infection and the host response to infection may be involved in the development of prostate cancer.

Studies that have investigated the role of infectious agents in the aetiology of prostate cancer have adopted single organism targeted approaches or have identified microbial constituents based on amplification of various hypervariable regions of the $16 \mathrm{~S}$ rRNA gene in concert with traditional cloning and sequencing methods [6-9]. Single organism targeted approaches are limited by their specificity while traditional broad-range 16S rRNA gene amplification, cloning and Sanger sequencing can be laborious and costly, depending on the scale of the study, number and complexity of samples. When compared with conventional sequencing methods, cyclic arraybased massively parallel sequencing (MPS) methods, albeit with shorter read length capability and less 
accuracy in base calling, offer efficiencies in terms of cost, time and scalability.

The principal hypothesis that guided the direction of the work presented in this study was that persistent, rather than transient, infection of the prostate gland by a sexually transmitted or other infectious agent would be associated with risk. Thus, evidence of infection at the tissue level was sought by utilising two different molecular approaches, targeted partial 16S rRNA gene sequencing and total RNA sequencing using MPS. The overall objective of this study was to investigate the presence of infectious agent(s) in histopathologically determined aggressive prostate cancer cases (Gleason score $\geq 8$ ).

\section{Methods}

\section{Samples}

Fresh-frozen scalpel-excised prostate tissue from males that had undergone radical prostatectomy with a Gleason score of $\geq 8$ and tumour stage ranging from pT2c to $\mathrm{pT} 3 \mathrm{~b}$ (inclusive) were obtained from the Australian Prostate Cancer Bioresource [10] $(n=10)$. Tumour and benign tissues were provided for each case and the presence/absence of malignant tissue was confirmed by histopathology by a single pathologist (JP).

\section{Nucleic acid extraction}

Frozen tissue was disrupted by freeze fracture, Buffer RLT Plus (Qiagen, Hilden, Germany) containing $\beta$ mercaptoethanol was added. The lysate was further homogenised using a QIAshredder ${ }^{\bullet}$ (Qiagen, Hilden, Germany) column and then underwent enzymatic digestion and nucleic acid extraction with the AllPrep DNA/RNA Mini Kit (Qiagen, Hilden, Germany) according to the manufacturer's instructions. Both DNA and RNA isolates were stored at $-80{ }^{\circ} \mathrm{C}$ (Additional file 1).

\section{Quantitative and qualitative assessment of extracted DNA and RNA}

The concentration and integrity of sample RNA was assessed with the Bioanalyzer 2100 instrument (Agilent Technologies) using RNA 6000 Nano Kit (Agilent Technologies). The concentration of sample DNA was assessed by Qubit ${ }^{\circ}$ 1.0 Fluorometer (Life Technologies, Carlsbad, California, USA) and the Qubit ${ }^{\circ}$ dsDNA BR Assay Kit (Life Technologies, Carlsbad, California, USA).

\section{Quantification, normalisation and pooling of libraries}

Each RNA sample was normalised to $100 \mu \mathrm{g} / \mu \mathrm{L}$ in UltraPure $^{\mathrm{Tm}}$ DNAse/RNAse-Free Distilled Water (Invitrogen $^{\mathrm{Th}}$, Burlington, USA). Normalised RNA samples were pooled in equimolar amounts according to tissue type i.e. "malignant" or "benign".
$16 \mathrm{~S}$ rRNA amplicon sequencing $16 S$ rRNA polymerase chain reaction

Each PCR reaction contained 1X GeneAmp ${ }^{\circ}$ PCR Buffer II (Roche Molecular Systems, Inc. Branchburg Township, USA), $10 \mu \mathrm{M}$ (each) forward and reverse primer, $0.1 \mathrm{U}^{\text {AmpliTaq Gold }}{ }^{\circ}$ DNA polymerase (ThermoFisher Scientific, Waltham, Massachusetts, USA), $2.5 \mu \mathrm{M}$ $\mathrm{MgCl}_{2}, 400 \mu \mathrm{M}$ dNTPs, $2 \mu \mathrm{L}$ of template DNA in a final volume of $20 \mu \mathrm{L}$ with UltraPure ${ }^{\text {Tx }}$ DNAse/RNAse-Free Distilled Water (Invitrogen ${ }^{\mathrm{Tw}}$, Burlington, USA). Amplification of each sample was performed in triplicate using a Veriti ${ }^{\circ}$ 96-well Thermal Cycler (Applied Biosystems, Forster City, CA, USA). Negative amplification controls included $\mathrm{dH}_{2} \mathrm{O}$ and TE buffer and the positive amplification control was Salmonella typhimurium $(0.5 \mathrm{ng} / \mu \mathrm{L})$. Cycling conditions were as follows: $95{ }^{\circ} \mathrm{C}$ for $5 \mathrm{~min}$, 35 cycles at $95{ }^{\circ} \mathrm{C}$ for $45 \mathrm{~s}, 56{ }^{\circ} \mathrm{C}$ for $60 \mathrm{~s}$ and $72{ }^{\circ} \mathrm{C}$ for $90 \mathrm{~s}$, with a final extension at $72{ }^{\circ} \mathrm{C}$ for five minutes.

\section{Primers}

Universal primers $101 \mathrm{~F} / 534 \mathrm{R}$ and $515 \mathrm{~F} / 806 \mathrm{R}$ were used to amplify the V2-V3 and V4 hypervariable region of the 16S rRNA gene, respectively. The $\mathrm{V} 4$ region primer constructs were taken from Caporaso et al. (2011) [11] (supplementary methods). The V2-V3 region primer constructs were modified from [11] using V2-V3 region specific primers [12] to target the 16S rRNA V2-V3 hypervariable region (Additional file 1). Reverse primers were barcoded to enable multiplexing of samples.

\section{Purification of $P C R$ products}

Replicate wells were combined for each sample and excess primers, primer dimers and extraneous products were removed using a double-sided size selection/cleanup with Agencourt $^{\circ}$ AMPure $^{\odot}$ XP beads (Beckman Coulter, Inc., Massachusetts, U.S.A). Purified product

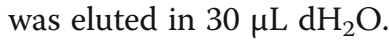

\section{Quantification and normalization of library pools}

Library size and quantity were assessed using the Bioanalyzer 2100 using the High Sensitivity DNA kit (Agilent Technologies Inc., Waldbronn, Germany). Individual samples were combined in equimolar quantities for sequencing.

\section{Sequencing}

Three custom primers were used for sequencing of the $16 \mathrm{~S}$ rRNA V4 region amplicons as described in [11] and the 16S rRNA V2-V3 region amplicons as adapted from Caporaso et al. (2011) [11]. The libraries were sequenced by using the $\mathrm{MiSeq}^{\circ} 500$ cycle Reagent Kit v2 (Illumina, Inc., San Diego, CA, USA). 


\section{Data analysis}

The quality of raw reads was assessed using FastQC v0.10.1 [13]. Paired-end reads were then stitched using FLASh (Fast Length Adjustment of Short reads) v1.2.6 [14] to generate full length reads of the of the sequenced amplicons. The quality of the FLASh-stitched reads were again assessed using FastQC v0.10.1 [13].

The QIIME (Quantitative Insights Into Microbial Ecology) pipeline and software package (version 1.7.0) [15] were used for data analyses using Closed-reference Operational Taxonomic Unit (OTU) picking. The sequences were clustered against a reference sequence collection [16] (Greengenes 12_10 reference collection) and any reads that did not hit a sequence at $97 \%$ sequence similarity to the reference sequence collection were excluded from downstream analysis.

\section{Total RNA/cDNA sequencing}

\section{Library preparation and sequencing}

Library preparation was performed using the Illumina ${ }^{\circ}$ TruSeq ${ }^{\circ}$ Stranded Total RNA Sample Preparation Kit in accordance with the manufacturer's instructions, however, did not include the initial poly(A) purification step (supplementary methods). The libraries were assessed with the Bioanalyser 2100 using the Bioanalyser DNA 1000 kit (Agilent). Individual libraries (tumour and cancer-unaffected prostate pools) were normalised to 2 $\mathrm{nM}$. Sequencing was performed on the HiSeq 2000.

\section{Data analysis}

Raw data underwent quality control and sequencing adapters were removed using Nesoni [17]. The full data set was queried for specific viral genomes (including human papillomaviruses 16 and 18, Herpes simplex virus 2 and Polyomaviruses) using human endogenous retroviruses (HERVs) as internal control as HERVs are remnant ancient retroviral sequences integrated into human germline DNA, some of which are actively transcribed. Reads were mapped to human rRNA (and other noncoding RNA) and to human mRNA using the SHort Read Mapping Package (SHRiMP) [18] and BurrowsWheeler Aligner (BWA) [19], respectively. Aligned reads were removed from the dataset. Unmapped reads were assembled into contiguous sequences using the de novo assembler Velvet [20], under kmer values of 55, 65, 75 and 85. The assemblies were queried with Easy-WebBLAST $+[21]$ for $16 \mathrm{~S}$ rRNA sequences and the presence of viral proteins (specifically all viral polymerases within the NCBI's RefSeq viral protein reference database [22]).

\section{Results}

\section{Characteristics of the case series}

The mean age at radical prostatectomy of patients was 64.5 years. Three cases underwent radical laparoscopic robotic prostatectomy while the remaining seven cases had open radical retropubic prostatectomy. All cases were considered to be of an aggressive nature and were selected on the basis of a Gleason score of $\geq 8$ and a TNM stage of at least PT2c (Table 1).

\section{S rRNA V4 hypervariable region}

One thousand three hundred and twenty four unique OTUs were identified in all 20 prostate tissue samples combined. Per sample, the mean number of OTUs present was 231.55 (SD 48.45) and ranged from 151 to 314. Community composition was reasonably complex.

Overall, the most abundant taxa identified were assigned to the family Enterobacteriaceae $(70.1 \%)$ and the genus Escherichia (6.9\%). There were five other unique OTUs that represented $\geq 1 \%$ of the microbial community observed across all samples. These taxa included Pseudomonadaceae (1.2\%), Comamonadaceae (1.2\%), Ralstonia (1.7\%), Pseudomonas (1.3\%) and Acinetobacter (1.1\%). There were five OTUs that represented $0.5<1 \%$ of the microbial community observed and these included Corynebacterium (0.8\%), Caulobacteriaceae (0.7\%), Curvibacter (0.7\%) Aerococcus (0.6\%) and Bradyrhizobium (0.6\%) The remaining 13.7\% of sequences were assigned to 308 other unique OTUs (Additional file 2).

The greatest proportion of sequences, ranging from 37.2 to $81.2 \%$, for each individual sample was represented by the family Enterobacteriaceae.. The prevalence of Escherichia ranged from 3.1 to $10.3 \%$ in the samples. Both taxa were represented in every sample. While there was up to a two-fold difference in the number of observed OTUs (151 to 314) among samples, the community composition of the most abundant samples (abundance $>0.5 \%$ ) was reasonably consistent across individual samples, however, some taxa including Pseudomonadaceae, Aerococcus, Corynebacterium and Actinobacter lwoffii were overrepresented in a number of samples when compared to their contribution to overall abundance (Additional file 2).

A group of 18 OTUs was found to be present in 95\% of samples (Table 2). While these 18 OTUs only represented a small proportion (on average 7.8\%) of the overall membership of prostatic microbial community, they contributed to a large proportion $(84.6 \%)$ of the relative abundance of the total communities of the 20 samples sequenced. The relative contribution of each 'core' OTU was reasonably consistent across samples (Fig. 1) with Enterobacteriacae (84.4\%) and Escherichia (8.3\%) the most abundant taxa contributing the 'core' community.

\section{S rRNA V2-V3 hypervariable region}

Six hundred and thirty four unique OTUs were present in all 20 prostate tissue samples combined. On a per 
Table 1 Histopathological features (Gleason score and TNM stage), age at radical prostatectomy and pre-operative PSA (ng/ $\mu \mathrm{L}$ ) for ten prostate cancer cases obtained from the Australian Prostate Cancer BioResource

\begin{tabular}{|c|c|c|c|c|c|}
\hline Patient ID & Gleason Score & TNM Stage & Age (years) at resection & Surgical type & Pre-operative PSA (ng/ $\mathrm{LL}$ ) \\
\hline PI & 8 & PT3ANO & 67.6 & Open & 26.7 \\
\hline P2 & 9 & РТ3В & 68.9 & Open & 6.2 \\
\hline P3 & 9 & PT3AN1MX & 73.3 & Open & 1.9 \\
\hline P4 & 9 & PT2CNO & 61.5 & Open & 3.1 \\
\hline P5 & 9 & PT2C & 59.2 & Robot & 5.7 \\
\hline P6 & 9 & PT3BNO & 64.4 & Robot & $\mathrm{n} / \mathrm{a}$ \\
\hline P7 & 8 & PT3ANO & 68.1 & Open & 13.9 \\
\hline P8 & 9 & РТЗА & 61.1 & Open & 9.2 \\
\hline P9 & 9 & PT3ANO & 53.4 & Open & $\mathrm{n} / \mathrm{a}$ \\
\hline P10 & 8 & PT3ANO & 67.8 & Robot & 8.8 \\
\hline
\end{tabular}

sample basis, the mean number of OTUs present was 117.95 (SD 23.95) and ranged from 71 to 160.

All samples combined, Enterobacteriaceae was dominant taxon (55.4\%), followed by Escherichia (20.9\%). There were seven additional OTUs with an abundance $\geq 1 \%$ including Comamonadaceae (1.8\%), Hyphomonadaceae (1.5\%), Pseudomonas (3.4\%), Corynebacterium (1.3\%), Tepidimonas (1.2\%), P. acnes (1.1\%) and Acinetobacter
(1.0\%). Ralstonia and Lutemonas represented 0.8 and $0.6 \%$ of the total microbial community, respectively. The remaining $11 \%$ of sequences comprised of the 194 other OTUs (Additional file 3).

The highest proportion of sequences for each individual sample was assigned to Enterobacteriaceae with an abundance ranging from 21.9 to $69.4 \%$ followed by Escherichia with an abundance ranging from 6.5 to

Table 2 Taxonomic assignments of the 18 OTUs present in 95\% of samples $(n=20)$ that underwent sequencing of the V4 hypervariable region of the $16 \mathrm{~S}$ rRNA gene and their relative abundance

Core OTUs shared by $95 \%$ of samples

Relative abundance of OTU within the total community

k_Bacteria; P_Proteobacteria; c_Gammaproteobacteria; O_Enterobacteriales; f_Enterobacteriaceae; g_; s_

k_Bacteria; p_Proteobacteria; c_Gammaproteobacteria; O_Enterobacteriales; f_Enterobacteriaceae; g_Escherichia; s

k_Bacteria; P_Proteobacteria; c_Betaproteobacteria; o_Burkholderiales; f_Oxalobacteraceae; g_Ralstonia; s_

k_Bacteria; P_Proteobacteria; c_Gammaproteobacteria; O_Pseudomonadales; f_Pseudomonadaceae; g_Pseudomonas; s_

k_Bacteria; P_Actinobacteria; c_Actinobacteria; o_Actinomycetales; f_Corynebacteriaceae; g_Corynebacterium; s_

k_Bacteria; P_Proteobacteria; c_Betaproteobacteria; o_Burkholderiales; f_Comamonadaceae; g_Curvibacter; s_

k_Bacteria; P_Proteobacteria; c_Alphaproteobacteria; o_Caulobacterales; f_Caulobacteraceae; g_; s_

k_Bacteria; P_Firmicutes; c_Bacilli; o_Lactobacillales; f_Aerococcaceae; g_Aerococcus; s_

k_Bacteria; P_Firmicutes; c_Bacilli; O_Bacillales; f_Staphylococcaceae; g_Staphylococcus; s_

k_Bacteria; P_Actinobacteria; c_Actinobacteria; O_Actinomycetales; f_Microbacteriaceae; g_; s_

k_Bacteria; P_Firmicutes; c_Bacilli; O_Bacillales; f_Staphylococcaceae; g_; s_

k_Bacteria; p_Proteobacteria; c_Gammaproteobacteria; o_Enterobacteriales; f_Enterobacteriaceae; g_Enterobacter; s_hormaechei

k_Bacteria; P_Proteobacteria; c_Gammaproteobacteria; O_Pseudomonadales; f_Moraxellaceae; g_; s_

k_Bacteria; P_Proteobacteria; c_Gammaproteobacteria; o_Enterobacteriales; f_Enterobacteriaceae; g_Plesiomonas; s_

k_Bacteria; P_Firmicutes; c_Bacilli; o_Lactobacillales; f_Streptococcaceae; g_Streptococcus; s_

k_Bacteria; P_Proteobacteria; c_Gammaproteobacteria; O_Enterobacteriales; f_Enterobacteriaceae; g_Erwinia; s_ 


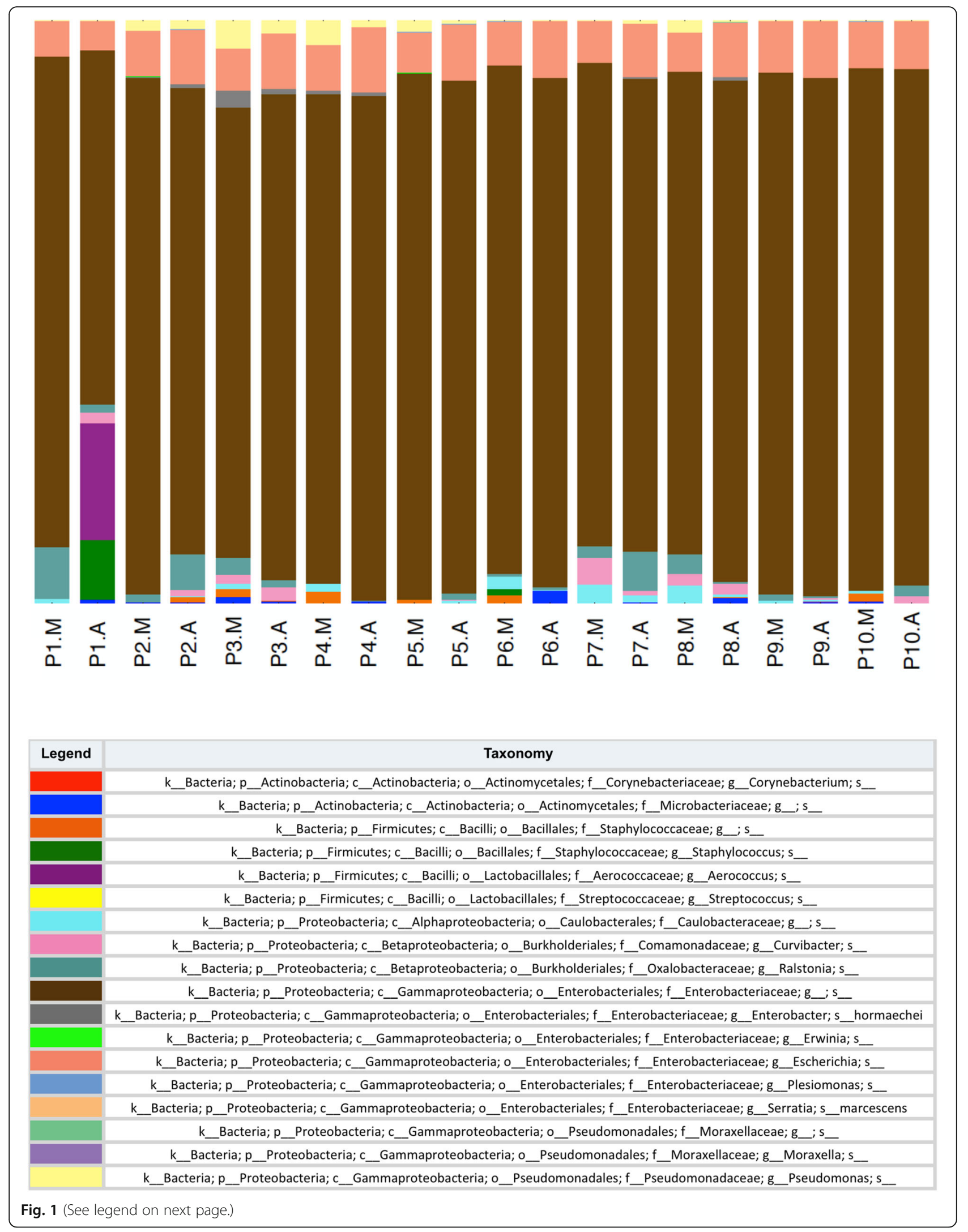


(See figure on previous page.)

Fig. 1 Taxa summary of 'core' OTUs identified in $95 \%$ of samples $(n=20)$ that underwent sequencing of the V4 hypervariable region of the 165 rRNA gene. The figure depicts the relative contribution of each member of the 'core' community to each sample in addition to its overall contribution to the core community over all samples combined. The contribution of taxa to the core community is expressed as a percentage. The letter A next to the patient ID denotes "adjacent" tissue and $\mathrm{M}$ denotes "malignant" tissue. The letters in the taxonomy column refer to $\mathrm{k}$ kingdom, $\mathrm{p}$ - phylum, c -class, o-order, f-family, $\mathrm{g}$ - genus, $\mathrm{s}$ - species

29.9\%. Both were represented in every sample. The contribution of the most abundant taxa $(>0.5 \%)$ to the community composition of each sample was reasonably consistent despite a two-fold difference in the number of observed OTUs (71 to 160). However, some taxa were overrepresented in a number of samples when compared to their contribution to overall abundance (Additional file 3).

Seven OTUs were represented in $95 \%$ of samples $(n=$ 20) and together they constituted the 'core' community within these prostate tissue samples (Table 3). These OTUs were assigned to Enterobacteriaceae and Streptococcaceae, Staphylococcus, Escherichia, Moraxella, Propionibacterium acnes and Streptococcus pseudopneumoniae. Despite these 'core' OTUs representing only a small proportion (on average 5.9\%) of the mean number of OTUs that comprise the overall prostatic microbial community, they contributed to a very large proportion (77.9\%) of the relative abundance of the total communities of the 20 samples sequenced. The relative contribution of each of the seven 'core' OTUs was reasonably consistent across individual samples (Fig. 2). Enterobacteriaceae and Escherichia were observed to be the most abundant taxa contributing to the 'core' community with a relative abundance of 72.2 and $26.6 \%$ respectively.

\section{Total RNA sequencing}

Human endogenous retroviral sequences (HERVs) were successfully detected in both benign and malignant datasets. After removing human ribosomal RNA and other non-coding read pairs, approximately 20 million read pairs remained for each of the malignant and benign prostate tissue datasets. Removing human mRNA left approximately 2.8 million unmapped read pairs for both the malignant and benign datasets. The unmapped reads were assembled into contiguous sequences using Velvet at kmer values of 55, 65, 75 and 85 and were queried for sequences of interest using BLAST. Sequences identified as belonging to Pseudomonas spp. were detected in the benign prostate tissue dataset. No sequences analogous to the NCBI RefSeq [22] library of viral polymerases (with the exception of HERVs) were detected. No specific viral sequences including human papillomaviruses, polyomaviruses, herpes simplex virus 1 and 2, were detected in either dataset.

\section{Discussion}

We used broad-range methods (one targeted and one agnostic) to explore and characterise microbial constituents within the prostate tissue of men with aggressive prostate cancer.

Previous studies have investigated the presence of bacterial, viral and prokaryotic organisms and their association with prostate cancer $[9,23,24]$ using other methodologies including traditional bacterial culture, specific, targeted PCR and bacterial 16S rRNA amplification, traditional cloning and capillary sequencing methods. The advantage of MPS, in this context, is the capacity to sequence the entire genomic/transcriptomic content of samples without a priori knowledge of specific genes and targets [25], in addition to its sensitivity

Table 3 Taxonomic assignments of the 7 OTUs present in 95\% of samples $(n=20)$ that underwent sequencing of the V2-V3 region of the 165 rRNA gene and their relative abundance 


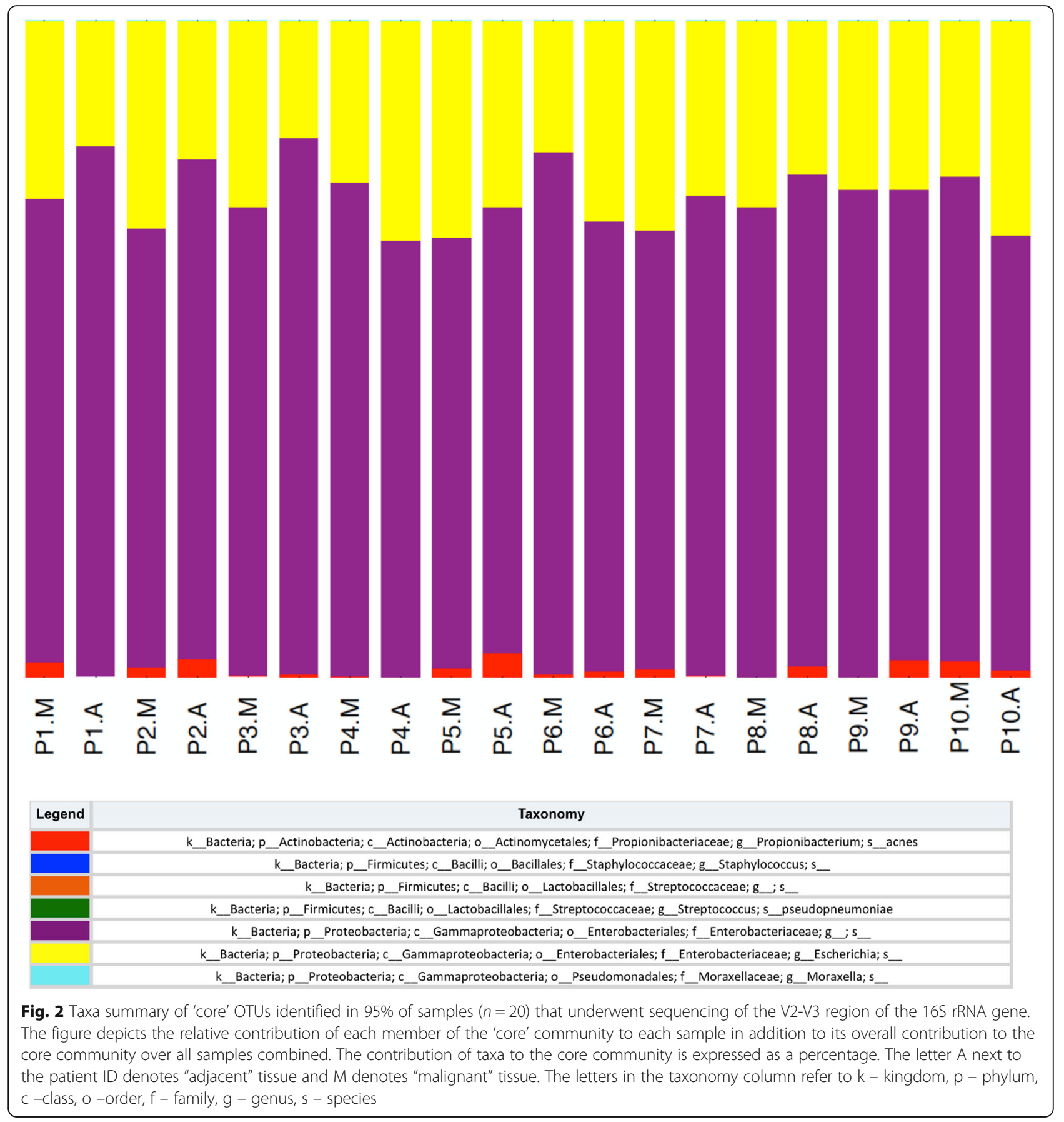

and high-throughput capability. However, despite the advantages of applying new technology to a decades-old question, the data generated and the methods used for data analysis were still in early development. As this field evolves, the methods, data, analytical tools and strategies will become more refined and enable further elucidation of these study questions.

To date, five studies $[8,9,26-28]$ have investigated and characterised bacterial 16S rRNA sequences in prostate tissue collected from prostate cancer patients. Only one of these studies [28] found no evidence of 16S rRNA sequences in prostate cancer tissues. Four studies $[8,9$, $26,27]$ demonstrated the presence of bacterial sequences in $88.9,85.7,19.6$ and $87 \%$ of patients, respectively. The most common organisms identified in these studies were members of the family Enterobacteriaceae and specifically species related to Escherichia coli. These findings are consistent with the results of the present study. In addition, analysis of the 16S rRNA V4 region sequencing data identified Actinobacter spp., Pseudomonas spp. and 
Streptococcus spp. as being present in $95 \%$ of all prostate samples therefore members of the 'core' community, in accordance with Sfanos et al. (2008). Analysis of the V2V3 region also identified Enterobacteriaceae, Escherichia spp. as the predominant taxa within this sample of prostate tissues in addition to Staphylococcus spp, Streptococcus spp, Moraxella spp., and Propionibacterium acnes as members of the 'core' community.

Distinguishing between contamination of tissue and 'true' prostatic microbial constituents is one of the main challenges of bacterial community studies. Studies $[8,27]$ have suggested that the presence of bacterial sequences in prostate cancer tissues reflects bacterial contamination of the prostate via transrectal prostate biopsy of prostate which is routinely performed to confirm a diagnosis of prostate cancer. This could explain the presence of bacterial 16S rRNA sequences in prostate tissue samples from prostate cancer patients and the range of organisms detected in our dataset also supports this hypothesis.

Catheterization of patients has also been suggested as a way in which the prostate may be contaminated with bacteria. Hochrieter et al. (2000) detected 16S rRNA sequences in all four prostate tissue samples taken from a benign prostatic hyperplasia $(\mathrm{BPH})$ patient that had an indwelling catheter for several weeks before radical prostatectomy [27]. Gorelick et al. (1988) performed quantitative bacterial culture of prostate tissues from prostatectomy patients to determine the prevalence of prostate bacterial infection or colonization [29]. They reported that $34 \%$ of patients with a pre-operative indwelling catheter returned a positive prostatic culture. Organisms were identified as common urinary tract pathogens including E. coli and Streptococcus fecalis. The pre-operative status with respect to catheterization of patients included in this study is unknown, however, it is a possibility that bacterial sequences identified in our samples could have been introduced in this way.

Sequences representing Propionibacterium acnes were detected in the V2-V3 16S rRNA dataset in 95\% of samples albeit at low abundance. This study reports a 95\% prevalence of $P$. acnes in prostate tissue samples which is consistent with the $100 \%$ prevelance of $P$. acnes detected in prostatic intraepithelial neoplasia (PIN) lesions and $78 \%$ of prostate cancer tissues reported by Fehri et al. (2011) but approximately two-fold higher than the prevalence of $P$. acnes reported by other studies $[1,2,9$, $30]$. The present study could not determine whether the $P$. acnes sequences detected in the V2-V3 dataset represented either urogenital or cutaneous strains. Therefore, it is difficult to ascertain if the P. acnes detected in these samples represent contamination through laboratory handling and reagents or if they have biological significance.
The study design and methods employed in this study had several limitations that may have diminished the ability to detect infectious organisms in prostate tissues that were of clinical significance. The study design employed to identify potential infectious agents associated with prostate cancer was limited by study sample collection methods, the sampling of prostate tissue, small sample size and sensitivity of detection (total RNA sequencing). In addition, there were inherent limitations to our study design including the presence of multiple 16S rRNA gene copies, extraction methods, library preparation, experimental controls and bioinformatics approaches.

The 16S rRNA gene occurs in at least one copy of every bacterial genome, however can also occur as multiple and heterogeneous copies with copy number ranging from 1 to 15 [31]. The E. coli genome contains seven copies of the 16S rRNA gene and the $P$. acnes genome three copies [32]. Most 16S rRNA gene surveys assume that the relative abundance of $16 \mathrm{~S}$ sequences are an accurate surrogate measure of the relative abundance of microorganisms in studies of community composition [31]. However, differences in the copy number/heterogeneity of the target $16 \mathrm{~S}$ rRNA gene may result in overestimation of diversity and abundance [33, 34]. Therefore, inferences made on the basis of relative abundance of 16S rRNA genes may not be an accurate representation of actual community composition [31, 35] and variation in $16 \mathrm{~S}$ rRNA gene copies can be a source of significant systemic bias within $16 \mathrm{~S}$ rRNA gene surveys [33]. This study did not normalize for variation in $16 \mathrm{~S}$ rRNA copy number and therefore it is unlikely that the reported relative abundances of taxa identified reflected actual taxa abundance. However, there are software tools [31] and a publicly available curated database (ribosomal RNA operon copy number database or rrnDB [35]) that could be applied to estimate actual organism abundance from $16 \mathrm{~S}$ rRNA gene abundance data in future work.

There is considerable scope to extend and improve upon the experimental design of this study in investigating a persistent infectious aetiology for prostate cancer. Incorporating a prospective study design that collected tissues specifically for PCR- and sequencing-based analyses may reduce the prevalence of contaminating sequences. Inclusion of (a) control group(s) that included samples from lower grade and less aggressive prostate cancer cases and cancer-unaffected prostates such as those from organ donors, cystoprostatectomy and/or $\mathrm{BPH}$ cases would allow comparison between the microbial constituents of different prostate pathologies (if any) and normal prostate tissue. In addition, a greater number of cases would ensure that the study is sufficiently powered to detect differences in microbial communities 
(if any) between groups. Sampling a greater proportion of the prostate gland at several anatomical sites would provide comprehensive coverage of the prostate gland as a whole. With regard to $16 \mathrm{~S}$ rRNA amplicon sequencing, the inclusion of extraction, PCR and water controls in sequencing runs would also provide a profile of laboratory contaminants so that 'true' microbial constituents (if any) could be distinguished from contaminating sequences. Normalization of $16 \mathrm{~S}$ rRNA datasets to account for heterogeneity of $16 \mathrm{~S}$ rRNA gene copies would also provide more accuracy with respect to relative organismal abundance. In terms of RNA sequencing, depletion of host RNA and enrichment of microbial rRNA and mRNA may increase detection sensitivity. If microorganisms of interest were detected, follow-up studies including verification of specific infectious agents in original nucleic acid samples via PCR and tissue localization studies would be warranted.

\section{Conclusions}

An infectious aetiology for prostate cancer has long been conjectured. We evaluated new technology to assess if its use could clarify the inconsistency in evidence related to the nature of possible infection(s) and their relationship to prostate tumour grade. We applied targeted and agnostic approaches both involving MPS. This technology detected endogenous retroviruses providing proof of concept but there was no clear evidence of clinically significant bacterial or viral sequences in prostate cancer tissue. As these investigative methods and protocols become more refined, MPS approaches are anticipated to have significant utility in identifying potential pathogens involved in disease aetiology. Further studies, specifically designed to detect associations between the disease phenotype and aetiological agents, are required.

\section{Additional files}

\section{Additional file 1: Supplementary methods. (DOCX $21 \mathrm{~kb}$ )}

Additional file 2: Complete 16 rRNA V4 region taxa summary. (DOCX $2335 \mathrm{~kb}$ )

Additional file 3: Complete 16 rRNA V2-V3 region taxa summary. (DOCX $1291 \mathrm{~kb}$ )

\section{Abbreviations}

cDNA: complementary DNA; COX-2: Cyclooxygenase-2;

DNA: Deoxyribonucleic acid; HERV: Human endogenous retrovirus; MPS: Massively parallel sequencing: mRNA: messenger RNA;

OTU: Operational taxonomic unit; PCR: Polymerase chain reaction; RNA: Ribonucleic acid; RNASEL: Ribonuclease L; rRNA: Ribosomal RNA;

SD: Standard deviation; TLR4: Toll-like receptor 4

\section{Acknowledgements}

Data analyses of the V2-V3 and V4 165 rRNA datasets were carried out by Gayle Philip, Life Sciences Computation Centre, Victorian Life Sciences Computation Initiative, University of Melbourne. Data analyses of the total RNA sequencing datasets were carried out by Dieter Bulach, Life Sciences
Computation Centre, Victorian Life Sciences Computation Initiative, University of Melbourne.

Amplification primers that targeted the V2-V3 hypervariable region of the $16 \mathrm{~S}$ rRNA gene were adapted/designed by Josef Wagner (JW), Murdoch Children's Research Institute.

The authors would like to express their appreciation to the study participants who kindly donated tissue to the Australian Prostate Cancer BioResource. The Australian Prostate Cancer BioResource is supported by the National Health and Medical Research Council of Australia Enabling Grant (no. 614296) and by a grant from the Prostate Cancer Foundation Australia.

\section{Funding}

This work was supported by the National Health and Medical Research Council (APP504702), the Prostate Cancer Foundation of Australia (projects YIG19 and PG2709) and the Austin Urology Research Foundation supported by the Urologists of the Austin Hospital, Melbourne, Victoria, Australia. The authors would like to express their appreciation to the study participants who kindly donated tissue to the Australian Prostate Cancer BioResource. The Australian Prostate Cancer BioResource is supported by the National Health and Medical Research Council of Australia Enabling Grant (no. 614296) and by a grant from the Prostate Cancer Foundation Australia.

\section{Availability of data and materials}

The datasets generated and analysed in the current study are available from the corresponding author on reasonable request.

\section{Authors' contributions}

GGG, GS and MS conceived, designed and successfully sought funding for the study. GGG was the principal investigator of the prostate study resources utilized. MS and ST coordinated, designed and supervised the molecular studies. MY carried out the laboratory-based work and drafted the manuscript. JP provided expert pathology review. APCB provided tissue samples. All authors read and approved the manuscript.

\section{Competing interests}

The authors declare that they have no competing interests.

\section{Consent for publication}

Not applicable.

\section{Ethics approval and consent to participate}

This research using human tissue was carried out in accordance with the Declaration of Helsinki and was granted ethics approval by the Human Ethics Sub-Committee, University of Melbourne, Victoria, Australia (ethics ID: 1135850).

\section{Author details}

${ }^{1}$ Genetic Epidemiology Laboratory, Department of Pathology, Faculty of Medicine, Dentistry and Health Sciences, University of Melbourne, VIC, Australia 3010. ${ }^{2}$ Department of Microbiology and Infectious Diseases, Royal Women's Hospital, Parkville, VIC, Australia 3052. ${ }^{3}$ Department of Obstetrics and Gynaecology, University of Melbourne, Parkville, VIC, Australia 3010. ${ }^{4}$ Murdoch Childrens Research Institute, Parkville, VIC, Australia 3052. ${ }^{5}$ Cancer Epidemiology and Intelligence Division, Cancer Epidemiology Centre, Cancer Council Victoria, Level 2, 615 St Kilda Road, Melbourne, VIC, Australia 3004.

${ }^{6}$ Centre for Epidemiology and Biostatistics, Melbourne School of Population and Global Health, University of Melbourne, Level 3, 207 Bouverie Street, Carlton, VIC, Australia 3053. 7Department of Surgery, University of Melbourne, Austin Health, 145 Studley Road, Heidelberg, VIC, Australia 3084. ${ }^{8}$ TissuPath, 92-96 Ricketts Road, Mount Waverley, VIC, Australia 3149. ' Human Genetics Foundation (HuGeF), Via Nizza, 52-10126 Torino, Italy. ${ }^{10}$ Australian Prostate Cancer BioResource, the Prostate Cancer Research Program, Department of Anatomy and Developmental Biology, Monash University, Melbourne, VIC, Australia 3800

Received: 29 August 2016 Accepted: 13 December 2016

Published online: 13 January 2017

\section{References}

1. Cohen RJ, Shannon BA, McNeal JE, Shannon T, Garrett KL. Propionibacterium acnes associated with inflammation in radical 
prostatectomy specimens: a possible link to cancer evolution? J Urol. 2005 173:1969-74.

2. Alexeyev OA, Marklund I, Shannon B, Golovleva I, Olsson J, Andersson C, et al. Direct visualization of Propionibacterium acnes in prostate tissue by multicolor fluorescent in situ hybridization assay. J Clin Microbiol. 2007:45:3721-8.

3. Panguluri RCK, Long LO, Chen W, Wang S, Coulibaly A, Ukoli F, et al. COX-2 gene promoter haplotypes and prostate cancer risk. Carcinogenesis. 2004;25:961-6.

4. Casey G, Neville PJ, Plummer SJ, Xiang Y, Krumroy LM, Klein EA, et al. RNASEL Arg462GIn variant is implicated in up to 13\% of prostate cancer cases. Nat Genet. 2002;32:581-3. Nature Publishing Group.

5. Chen Y-C, Giovannucci E, Lazarus R, Kraft P, Ketkar S, Hunter DJ. Sequence variants of Toll-like receptor 4 and susceptibility to prostate cancer. Cancer Res. 2005;65:11771-8.

6. Riley DE, Berger RE, Miner DC, Krieger JN. Diverse and related 16S rRNAencoding DNA sequences in prostate tissues of men with chronic prostatitis. J Clin Microbiol. 1998;36:1646-52.

7. Alexeyev O, Bergh J, Marklund I, Thellenberg-Karlsson C, Wiklund F, Grönberg $\mathrm{H}$, et al. Association between the presence of bacterial 16S RNA in prostate specimens taken during transurethral resection of prostate and subsequent risk of prostate cancer (Sweden). Cancer Causes Control. 2006;17:1127-33.

8. Krieger JN, Riley DE, Vesella RL, Miner DC, Ross SO, Lange PH. Bacterial dna sequences in prostate tissue from patients with prostate cancer and chronic prostatitis. J Urol. 2000;164:1221-8.

9. Sfanos KS, Sauvageot J, Fedor HL, Dick JD, De Marzo AM, Isaacs WB. A molecular analysis of prokaryotic and viral DNA sequences in prostate tissue from patients with prostate cancer indicates the presence of multiple and diverse microorganisms. Prostate. 2008;68:306-20.

10. Australian Prostate Cancer Bioresource [Internet]. apcbioresource.org.au. [cited 2016 Jun 7]. Available from: https://www.apcbioresource.org.au.

11. Caporaso JG, Lauber CL, Walters WA, Berg-Lyons D, Lozupone CA, Turnbaugh PJ, et al. Colloquium Paper: Global patterns of 16S rRNA diversity at a depth of millions of sequences per sample. Proc Natl Acad Sci. 2011; 108 Suppl 1:4516-22.

12. Sundquist A, Bigdeli S, Jalili R, Druzin ML, Waller S, Pullen KM, et al. Bacterial flora-typing with targeted, chip-based Pyrosequencing. BMC Microbiol. 2007;7:108.

13. FastQC v0.10.1 [Internet]. bioinformatics.bbsrc.ac.uk. [cited 2016 Jun 7]. Available from: http://www.bioinformatics.bbsrc.ac.uk/projects/fastqc.

14. Magoč T, Salzberg SL. FLASH: fast length adjustment of short reads to improve genome assemblies. Bioinformatics. 2011;27:2957-63.

15. Caporaso JG, Kuczynski J, Stombaugh J, Bittinger K, Bushman FD, Costello EK, et al. QIIME allows analysis of high-throughput community sequencing data. Nat Meth. 2010;7:335-6.

16. Greengenes [Internet]. greengenes.lbl.gov. [cited 2016 Jun 7]. Available from: http://greengenes.lbl.gov/cgi-bin/nph-index.cgi.

17. Nesoni [Internet]. https://github.com/Victorian-Bioinformatics-Consortium/ nesoni [cited 2016 Oct 19]. Available from: https://github.com/VictorianBioinformatics-Consortium/nesoni.

18. David M, Dzamba M, Lister D, Ilie L, Brudno M. SHRiMP2: sensitive yet practical SHort Read Mapping. Bioinformatics. 2011:27:1011-2.

19. Li H, Durbin R. Fast and accurate short read alignment with BurrowsWheeler transform. Bioinformatics. 2009:25:1754-60.

20. Zerbino DR. Using the Velvet de novo assembler for short-read sequencing technologies. Curr Protoc Bioinformatics. 2010;Chapter 11:Unit11.5.

21. Easy-Web-BLAST+ [Internet]. github.com. [cited 2016 Jun 7]. Available from: https://github.com/tseemann/easy-web-blast.

22. NCBI RefSeq [Internet]. ftp.ncbi.nlm.nih.gov. [cited 2016 Jun 7]. Available from: ftp://ftp.ncbi.nlm.nih.gov/refseq/release/viral/.

23. Sutcliffe S, Viscidi RP, Till C, Goodman PJ, Hoque AM, Hsing AW, et al. Human papillomavirus types 16, 18, and 31 serostatus and prostate cancer risk in the Prostate Cancer Prevention Trial. Cancer Epidemiol Biomarkers Prev. 2010;19:614-8.

24. Sutcliffe S, Alderete JF, Till C, Goodman PJ, Hsing AW, Zenilman JM, et al. Trichomonosis and subsequent risk of prostate cancer in the Prostate Cancer Prevention Trial. Int J Cancer. 2009;124:2082-7.

25. Metzker ML. Sequencing technologies - the next generation. Nat Rev Genet. 2010;11:31-46.

26. Keay S, Zhang CO, Baldwin BR, Alexander RB. Polymerase chain reaction amplification of bacterial 16s rRNA genes in prostate biopsies from men without chronic prostatitis. Urology. 1999;53:487-91.
27. Hochreiter WW, Duncan JL, Schaeffer AJ. Evaluation of the bacterial flora of the prostate using a 165 rRNA gene based polymerase chain reaction. J Urol. 2000;163:127-30.

28. Leskinen MJ, Rantakokko-Jalava K, Manninen R, Leppilahti M, Marttila T, Kylmälä $T$, et al. Negative bacterial polymerase chain reaction (PCR) findings in prostate tissue from patients with symptoms of chronic pelvic pain syndrome (CPPS) and localized prostate cancer. Prostate. 2003;55:105-10.

29. Gorelick Jl, Senterfit LB, Vaughan ED. Quantitative bacterial tissue cultures from 209 prostatectomy specimens: findings and implications. J Urol. 1988;139:57-60.

30. Mak TN, Fischer N, Laube B, Brinkmann V, Metruccio MME, Sfanos KS, et al. Propionibacterium acnes host cell tropism contributes to vimentin-mediated invasion and induction of inflammation. Cell Microbiol. 2012;14:1720-33.

31. Kembel SW, Wu M, Eisen JA, Green JL. Incorporating 165 gene copy number information improves estimates of microbial diversity and abundance. PLoS Comput Biol. 2012;8:e1002743.

32. Shannon BA, Cohen RJ, Garrett KL. Influence of $16 \mathrm{~S}$ rDNA primer sequence mismatches on the spectrum of bacterial genera detected in prostate tissue by universal eubacterial PCR. Prostate. 2008;68:1487-91.

33. Acinas SG, Marcelino LA, Klepac-Ceraj V, Polz MF. Divergence and redundancy of $16 \mathrm{~S}$ rRNA sequences in genomes with multiple rrn operons. J Bacteriol. 2004;186:2629-35.

34. Jonasson J, Olofsson M, Monstein H-J. Classification, identification and subtyping of bacteria based on pyrosequencing and signature matching of 165 rDNA fragments. APMIS. 2002;110:263-72.

35. Stoddard SF, Smith BJ, Hein R, Roller BRK, Schmidt TM. rrnDB: improved tools for interpreting rRNA gene abundance in bacteria and archaea and a new foundation for future development. Nucleic Acids Res. 2015;43:D593-8.

\section{Submit your next manuscript to BioMed Central and we will help you at every step:}

- We accept pre-submission inquiries

- Our selector tool helps you to find the most relevant journal

- We provide round the clock customer support

- Convenient online submission

- Thorough peer review

- Inclusion in PubMed and all major indexing services

- Maximum visibility for your research

Submit your manuscript at www.biomedcentral.com/submit
) Biomed Central 